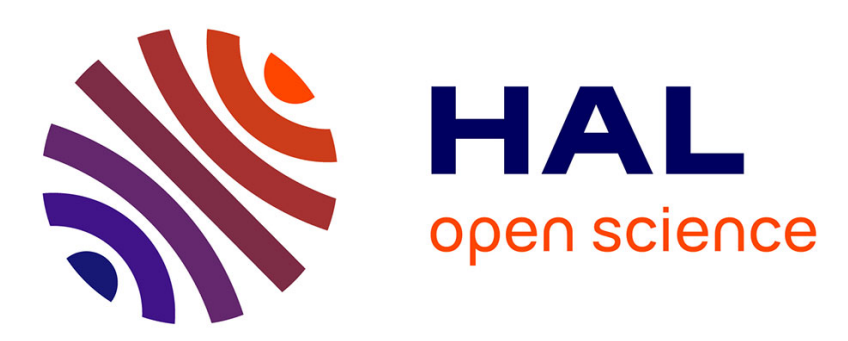

\title{
Étude optique des lames stratifiées d'hémine déposées sur support solide
}

\author{
R.G. Rinaldi, Stéphane Rosi
}

\section{To cite this version:}

R.G. Rinaldi, Stéphane Rosi. Étude optique des lames stratifiées d'hémine déposées sur support solide. J. Phys. Radium, 1956, 17 (12), pp.997-1000. 10.1051/jphysrad:019560017012099700 . jpa-00235606

\section{HAL Id: jpa-00235606 https://hal.science/jpa-00235606}

Submitted on 1 Jan 1956

HAL is a multi-disciplinary open access archive for the deposit and dissemination of scientific research documents, whether they are published or not. The documents may come from teaching and research institutions in France or abroad, or from public or private research centers.
L'archive ouverte pluridisciplinaire HAL, est destinée au dépôt et à la diffusion de documents scientifiques de niveau recherche, publiés ou non, émanant des établissements d'enseignement et de recherche français ou étrangers, des laboratoires publics ou privés. 


\title{
ÉTUDE OPTIQUE DES LAMES STRATIFIEES D'HÉMINE DEPOSEES SUR SUPPORT SOLIDE
}

\author{
Par Renaud RINALDi et Stéphane ROSI, \\ Laboratoire de Physique Générale de la Faculté des Sciences de Marseille. \\ Laboratoire de Physique Biologique de l'Université de Grenoble.
}

\begin{abstract}
Sommaire. - L'étude optique des lames minces formées par la superposition des couches monomoléculaires d'hémine en nombre croissant montre que ces couches non comprimées n'ont pas une structure continue et homogène tandis que l'état lacunaire des lames formées de couches comprimées est réduit par la compression.

Les courbes représentant les facteurs de réflexion et de transmission en fonction de l'épaisseur dans le cas des couches comprimées ont alors l'allure de celles que donnent les couches métalliques absorbantes continues et homogènes.
\end{abstract}

Généralités. -- Dans des travaux précédents [1] et [2] l'un de nous a montré, en collaboration avec P. Dubouloz, que l'étude optique des couches monomoléculaires donnait des résultats intéressants si l'on s'adressait à des couches formées par un corps organique absorbant.

Nous avons choisi comme premier matériel d'étude, l'hémine, pigment de constitution bien connue, qui présente quel que soit le solvant une bande d'absorption vers $4000 \AA$ et donne facilement à la surface de l'eau des couches monomoléculaires du type solide [3].

Une étude précédente [4] avait permis de mesurer en incidence normale, pour une série de douze lames d'épaisseur variant de une à douze couches monomoléculaires, les facteurs de réflexion $R$ de la lame dans l'air et $R^{\prime}$ de la lame dans le verre, ainsi que le facteur de transmission $T$ et les absorptions $A$ et $A^{\prime}$ respectivement définies lorsque la lumière vient $d u$ côté air par la relation :

$$
A=1-R-T
$$

et lorsqu'elle vient du côté support par :

$$
A^{\prime}=1-R^{\prime}-T \text {. }
$$

Outre la mise en évidence dè la bande d'absorption dans le violet, cette étude préliminaire a montré que, pour une longueur d'onde déterminée, les courbes représentatives des facteurs de réflexion et de transmission, en fonction de l'épaisseur des lames, présentaient des oscillations que nous avons alors essentiellement attribuées à l'état lacunaire des couches.

Nous nous sommes proposés ici de reprendre ce travail en essayant d'obtenir des lames plus homogènes en modifiant par compression la surface à déposer, et d'étudier des lames formées par un plus grand nombre de couches. Les résultats obtenus devraient alors se rapprocher de ceux que donne l'étude des lames métalliques minces [5], puisque le coefficient d'absorption atteint par l'hémine au voisinage du maximum est de l'ordre de grandeur des coefficients d'absorption métalliques.
Obtention des lames. - L'hémine peut être considérée comme le chlorure de l'hématine qui, avec la globine, constitue le pigment du sang. La présence de deux groupements carboxyles confère à la molécule une polarité quil l'oriente à la surface de l'eau ; tous les autres groupements étant en effet hydrophobes, la couche monomoléculaire superficielle sera constituée par des molécules littéralement fichées dans l'eau par les deux chaînes propioniques.

Insoluble dans l'eau, peu ou très peu soluble dans les solvants organiques, l'hémine est très soluble dans la pyridine avec laquelle elle donne une combinaison.

Le earactère polaire de la molécule lui permettant de donner facilement à la surface de l'eau une couche monomoléculaire de type solide, il est aisé d'amener cette couche à se déposer par écoulement sur un support transparent et d'en étudier les propriétés optiques.

En répétant plusieurs fois la même opération, on peut réaliser une lame d'hémine qui se présente comme un milieu stratifié comportant un grand nombre de couches monomoléculaires superposées.

La mesure par A. E. Alexander [3] de la surface moléculaire de l'hémine, qui est de $68 \AA^{2}$, permet de préparer une solution dont un volume connu, déposé à la surface de l'eau, donne une couche de surface déterminée. Le solvant est un mélange pyridine-benzène (1 pour 4) ce dernier corps facilite l'expansion de la couche qui s'étale sur l'eau à cause de sa faible solubilité et de son caractère polaire.

Le support de verre légèrement prismatique est placé horizontalement dans un cristallisoir que l'on remplit d'eau distillée à ras bord. La surface de l'eau est ensuite nettoyée au moyen a'une feuille de papier de soie posée à plat et retirée tangentiellement. Une boucle de soie paraffinée placée sur l'eau délimite à la surface de celle-ci une aire donnée ; il suffit alors de déposer à l'intérieur de la boucle une quantité déterminée de solution d'hëmine.

Par élimination du solvant (évaporation du ben- 
zène et dissolution de la pyridine) il reste à la surface de l'eau une lame monomoléculaire d'hémine de forme circulaire, isolée de la paroi et bien délimitée.

Afin de réduire les lacunes, on comprime la couche en déposant entre le fil de soie et le cristallisoir une petite quantité d'acide oléique qui se répand et exerce sur le pourtour de la couche une pression uniforme d'environ 30 dynes $/ \mathrm{cm}$. Le bord de la couche est déformé et la surface réduite d'environ $5 \%$ avec des solutions fraîches. La couche monomoléculaire une fois organisée, on abaisse très lentement le niveau de l'eau afin qu'elle se dépose sur le support de verre La présence de petites lentilles d'acide oléique à la surface de l'eau assure, lors de l'écoulement, une pression constante en remplaçant les molécules d'acide oléique qui s'accrochent aux parois.

En répétant plusieurs fois cette opération sur le même support, on obtient un milieu stratifié formé par la superposition de couches monomoléculaires d'hémine. Nous avons ainsi réalisé deux séries de lames : l'une formée de lames de une à vingt couches uniquement cerclées, l'autre constituée par dés lames comprenant de une à soixante couches monomoléculaires comprimées.

Mesures. - Pour chacune des lames de ces deux séries et pour vingt-deux longueurs d'onde couvrant le spectre de 3760 à $6250 \AA$, nous avons déterminé, en incidence normale, les facteurs de réflexion et de transmission $R, R^{\prime}$ et $T$, puis calculé les facteurs d'absorption $A$ et $A^{\prime}$.

Le spectrophotomètre utilisé, décrit par P. BousQuet [6], se compose d'une source stabilisée (lampe à filament de tungstène) suivie d'un monochromateur double à optique de quartz et d'unè cellule photoélectrique à multiplicateurs d'électrons (montage de Lenouvel).

Le support sur lequel on dépose la couche est une lame de verre légèrement prismatique (environ $3^{\circ}$ ) ce qui, en les séparant, permet d'utiliser, selon la mesure envisagée, les faisceaux réfléchis sur l'une ou l'autre des faces du support.

A cet effet, le prisme dont l'arête est verticale, est porté par un châssis métallique monté sur glissières, permettant de déplacer la face utile dans un plan vertical. Après chaque dépôt, la moitié supérieure de la lame de verre est nettoyée afin.de pouvoir comparer par déplacement du châssis, le faisceau intéressant la couche à celui qui n'a rencontré que le verre nu.

Les mesures des valeurs̀ $\alpha_{1}$ et $\alpha_{2}$ des déviations du spot, proportionnelles aux flux comparés $\Phi_{\mathrm{L}}$ et $\Phi_{\mathrm{V}}$ ayant respectivement rencontré la lame et le verre nu, permettent de déduire les valeurs relatives des facteurs de réflexion et de transmission de la lame mince.

En effet, si $\Phi_{0}$ est le flux incident, $\alpha$ la valeur du rapport $\alpha_{1} / \alpha_{2}, R_{\mathrm{v}}$ le facteur de réflexion du verre nu et $A_{\mathrm{V}}$ le facteur d'absorption du support, on a :

10 Pour $R$, facteur de réflexion de la lame mince côté air (fig. 1)

$$
\Phi_{\mathrm{L}}=\Phi_{0} R ; \Phi_{\mathrm{V}}=\check{\Phi}_{0} R_{\mathrm{V}} ; \alpha=\frac{\Phi_{\mathrm{L}}}{\Phi_{\mathrm{V}}}=\frac{R}{R_{\mathrm{V}}} \text { et } R={ }^{-} \alpha R_{\mathrm{V}} .
$$
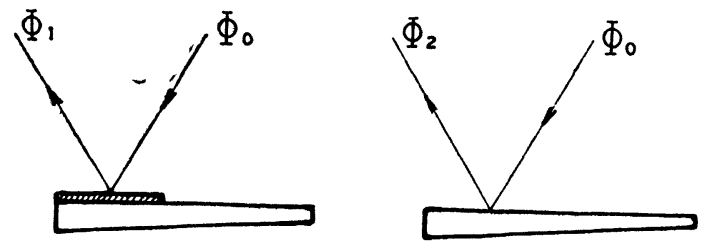

Fig. 1. - Mesure du facteur de réflexion côté air (pour la clarté du dessin les incidences n'ont pas été prises normales).

$2^{\circ}$ Pour $R^{\prime}$, facteur de réflexion.de la lame mince côté verre ( $f$ ig. 2)

$$
\begin{gathered}
\Phi_{\mathrm{L}}=\Phi_{0}\left(1-R_{\mathrm{V}}\right)\left(1-A_{\mathrm{V}}\right) R^{\prime}\left(1-A_{\mathrm{V}}\right)\left(1-R_{\mathrm{V}}\right) \\
=\Phi_{0} R^{\prime}\left(1-R_{\mathrm{V}}\right)^{2}\left(1-A_{\mathrm{V}}\right)^{2} \\
\begin{array}{c}
\Phi_{\mathrm{V}}=\Phi_{0}\left(1-R_{\mathrm{V}}\right)\left(1-A_{\mathrm{V}}\right) R_{\mathrm{V}}\left(1-A_{\mathrm{V}}\right)\left(1-R_{\mathrm{V}}\right) \\
=\Phi_{0} R_{\mathrm{V}}\left(1-R_{\mathrm{V}}\right)^{2}\left(1-A_{\mathrm{V}}\right)^{2}
\end{array} \\
\alpha^{\prime}=\frac{\Phi_{\mathrm{L}}}{\Phi_{\mathrm{V}}}=\frac{R^{\prime}}{R_{\mathrm{V}}} \text { et } R^{\prime}=\alpha^{\prime} R_{\mathrm{V}} .
\end{gathered}
$$
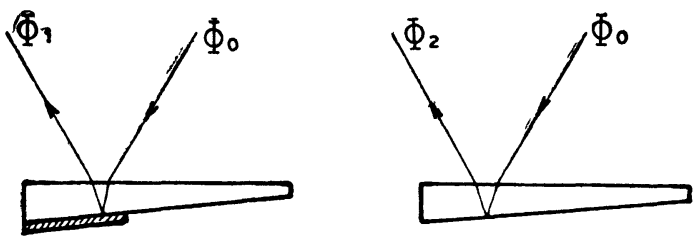

Fig. 2. - Mesure du facteur de réflexion côté support.

$3^{0}$ Pour $T$, facteur de transmission de la couche mince (fig. 3).

$$
\begin{gathered}
\Phi_{\mathrm{L}}=\Phi_{0} T\left(1-A_{\mathrm{V}}\right) R_{\mathrm{V}}\left(1-A_{\mathrm{V}}\right) T=\Phi_{0} T^{2} R_{\mathrm{V}}\left(1-A_{\mathrm{V}}\right)^{2} \\
\Phi_{\mathrm{V}}=\Phi_{0} R_{\mathrm{V}}\left(1-R_{\mathrm{V}}\right)^{2}\left(1-A_{\mathrm{V}}\right)^{2} \\
\alpha=\frac{\Phi_{\mathrm{L}}}{\Phi_{\mathrm{V}}}=\frac{T^{2}}{\left(1-R_{\mathrm{V}}\right)^{2}} \text { et } T^{2}=\alpha\left(1-R_{\mathrm{V}}\right)^{2} .
\end{gathered}
$$
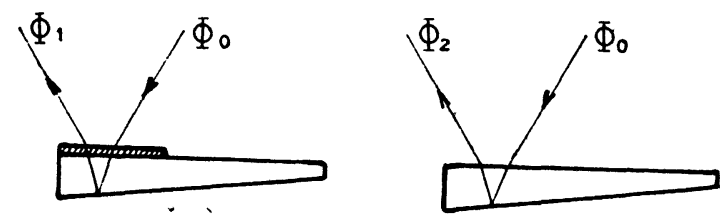

Fig. 3. - Mesure du facteur de transmission

La comparaison d'un flux réfléchi sur le verre utilisé et sur une surface de quartz, le facteur de réflexion du quartz étant calculable, permet de déterminer le facteur de réflexion' $\boldsymbol{R}_{\mathrm{v}} \mathrm{du}$ verre utilisé et par là même le facteur de réflexion absolu des lames. Les facteurs de réflexion et de trans. mission sont ainsi déterminés à 2 ou $3 \cdot 10^{-3}$ près. 
Résultats des mesures. - I. Couches non comPRimées. - Cette série, qui a comporté des lames successives formées de une à vingt couches superposées, met en évidence une bande d'absorption (fig. 4), dès la première couche, pour une longueur d'onde de $4000 \AA$. Les courbes représentant les

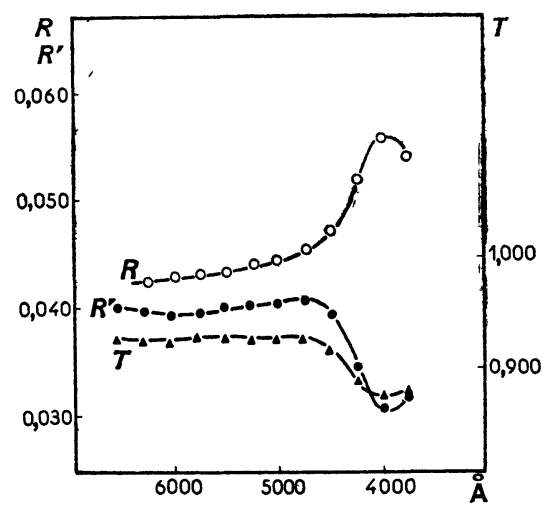

FIG. 4. - Lames non comprimées.

Variations de $R, R^{\prime}, T$, en fonction de la longueur d'onde pour une lame de 9 couches d'épaisseur.

différents facteurs, $R, R^{\prime}$ et $T$ varient peu d'une lame à l'autre et rappellent celles du travail précité [4] aux ordonnées près.

En étudiant les différents facteurs en fonction du nombre de couches, on note (fig. 5) que les

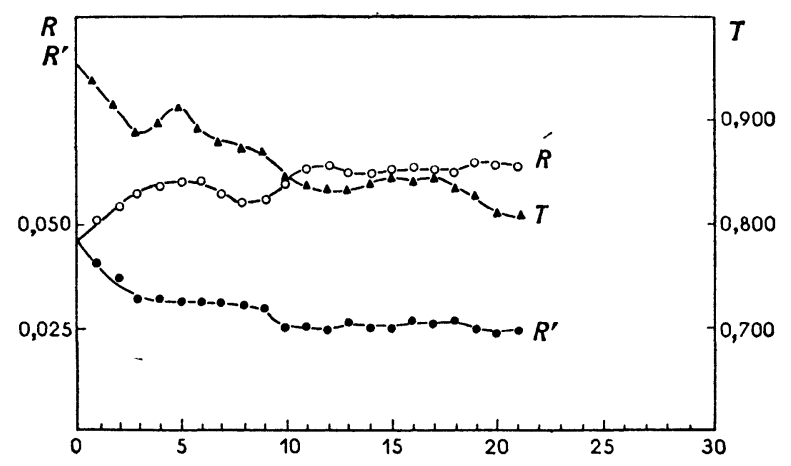

Fig. 5. - Lames non comprimées.

Facteurs de réflexion côté air $R$ côté support $R^{\prime}$ et transmission $T$, en fonction du nombre de couches pour la longueur d'onde $4000 \AA$.

oscillations présentées par les courbes donnant $R$, $R^{\prime}$ et $T$ en fonction des épaisseurs, sont légèrement décalées et beaucoup moins apparentes que celles obtenues précédemment [4], lorsque le fil de soie n'isolait pas la lame et que des pertes d'hémine sur les parois pouvaient alors se produire lors de l'écoulement. Ces oscillations, que nous avions attribuées à l'état lacunaire des lames, étant plus aplaties, et les facteurs de réflexion du côté air étant nettement plus élevés, on peut penser que dans le cas présent les couches sont plus homogènes. De plus, le fait que ces oscillations paraissent s'amortir à partir de la treizième lame peut laisser supposer que l'ensemble des couches forme déjà, par compensation, un milieu plus régulièrement homogène nous rapprochant de ceux que l'on réalise avec les lames métalliques.

II. Lames comprimées. - Pour les premières lames, les courbes donnant $R, R^{\prime}$ et $T$ ont la même allure que celles relatives aux couches non comprimées (fig. 6). Elles en diffèrent toutefois par des

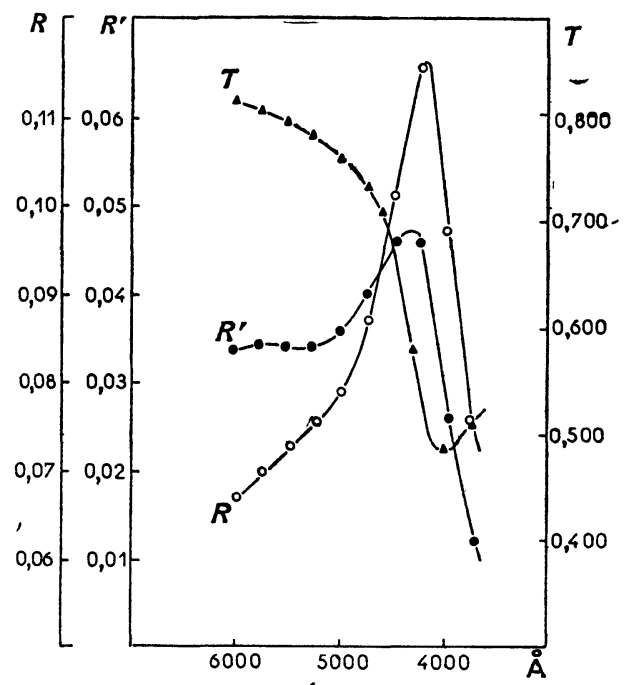

Fig. 6. - Lames comprimées.

Variations de $R, R^{\prime}, T$ en fonction de la longueur d'onde pour une lame de 21 couches d'épaisseur.

valeurs absolues différentes pour $R$ et $R^{\prime}$, par un maximum ou un minimum beaucoup plus accusé et par le fait que les courbes sont nettement décalées lorsqu'on passe d'une lame à la suivante. A mesure que le nombre de couches augmente, non seulement les courbes se resserrent, mais leur forme varie et les maxima et minima se déplacent. Ainsi, pour le facteur de réflexion côté air $R$, le maximum croît et passe de $4000 \AA$ pour les premières couches à $4250 \AA$ pour la $21 \dot{0}$, tandis que sa valeur diminue ensuite rapidement. Pour le facteur de réflexion côté support $R^{\prime}$, le minimum est également plus accusé, se déplace vers les courtes longueurs d'onde (nettement au-dessous de $3750 \AA$ pourla $10^{\circ}$ couche) alors qu'un sommet apparait pour la $10^{\circ}$ couche vers $4500 \AA$, croît jusqu'à la $31^{\circ}$ couche en se déplaçant vers les courtes longueurs d'onde puis décroît ensuite en revenant vers son point de départ. On constate également qu'entre la $31^{\mathrm{e}}$ et la $38 \mathrm{e}$ couche, $R^{\prime}$ continue à augmenter pour les grandes longueurs d'onde tandis qu'il diminue pour les petites, ce qui amène pour ces lames un pivotement des courbes autour de $5000 \AA$. Enfin, 
pour $T$, les courbes sont beaucoup plus régulières et gardent la même allure avec un minimumimmobile.

Pour étudier les facteurs de réflexion et de transmission en fonction du nombre de couches, nous avons tracé les courbes relatives aux longueurs d'onde suivantes :

$$
3730-4000-4250-4600-4950-5450 \AA
$$

dont les trois premières correspondent à la bande d'absorption sont les plus caractéristiques. Nous avons représenté ( $f i g$. 7) celle qui correspond

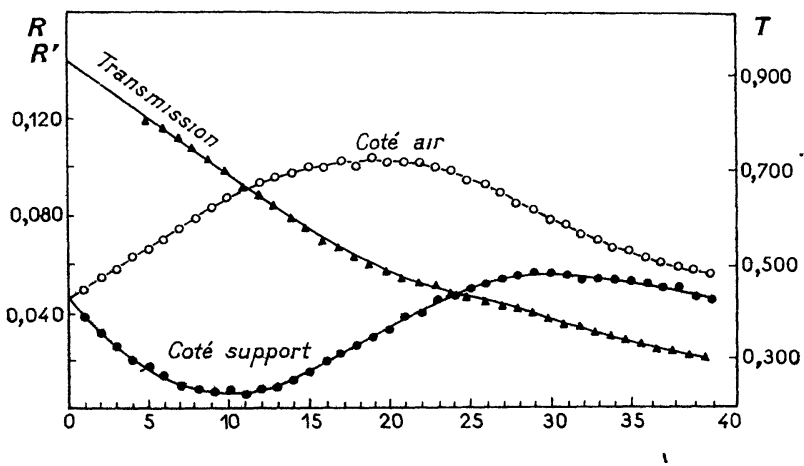

FIG. 7. - Lames comprimées.

Facteurs de réflexion côté air, côté support, et transmission en fonction du nombre de couches vour la longueur d'onde $4000 \AA$.

à $4000 \AA$, les autres sont semblables, mais le maximum de $R$ est obtenu pour un nombre de couches variable avec la longueur d'onde et correspond à 40 couches pour $5450 \AA$. Aucune de ces courbes ne fait apparaître d'oscillation et leur évolution est comparable à celle des lames métalliques absorbantes. L'absorption ne paraît pas varier régulièrement avec le nombre de couches jusqu'à la $8 \mathrm{e}$, puis les courbes très régulières qui sont presque des droites jusqu'à la 40 e couche, s'incurvent ensuite légèrement vers le bas.

Conclusions. - Cette étude semble faire apparaître deux ordres de faits :

$1^{0}$ Les couches monomoléculaires non com- primées, bien qu'elles soient parfaitement isolées par un cerclage, et par conséquent non soumises aux tractions accidentelles, se comportent optiquement, comme des couches non isolées. Si nous attribuons ce résultat au manque d'homogénéité des couches, nous sommes en accord avec l'étude du potentiel de surface sur le fait que l'idée de couches monomoléculaires solides n'implique pas forcément celle d'une structure continue et homogène [3]. Ces couches peuvent en effet être composées par une association de "plaques solides » séparées et formées elles-mêmes de molécules jointives.

Lors du dépôt sur le support solide les forces de traction qui s'exercent alors sur la couche monomoléculaire ne peuvent qu'accentuer cet état fragmentaire, favorisant ainsi cette structure lacunaire avec laquelle semble s'accorder nos résultats expérimentaux.

$2^{\circ}$ Dans le cas des lames formées par la superposition des couches comprimées, les courbes représentant les facteurs de réflexion et de transmission en fonction de l'épaisseur ne présentent plus d'oscillations et ont l'allure de celles que donnent les couches métalliques continues et absorbantes. Ceci paraît être dû au fait que la pression exercée a réduit les lacunes en serrant les unes contre les autres les molécules déjà orientées sur l'eau. On a sans doute dans ce cas un état analogue aux "closed packed chaines " d'Harkins et lors du dépôt la lame se découpe, par glissement des molécules sans que les forces de traction puissent provoquer des lacunes. En somme, l'état lacunaire des couches obtenues précédemment, et que l'on pouvait attribuer d'une part à des causes'accidentelles trouvant leur origine dans l'étirement de ces derniers lors du dépôt par accrochage sur les parois et, d'autre part, à la constitution même de la couche, se trouve réduit respectivement par cerclage qui isole les couches des parois, et par compression. Il paraît donc logique, pour les mesures à envisager à l'avenir, d'opérer sur des couches cerclées et comprimées, en raison de leur comportement comme des lames homogènes et continues.

Manuscrit reçu le 16 avril 1956.

\section{BIBLIOGRAPHIE}

[1] Dubouloz et Rinaldi, Revue d'Optique, 1954, 33, 630. [2] Rinaldi et Dubouloz, C. R. Acad. Sc., 1955, 240, 1697-1700.

[3] Alexander (A. E.), J. Chem. Soc., 1937, 2, 1813.

[4] Rinaldi, C. R. Acad. Sc., 1955, 240, 61-64.
[5] Rouard, Propriétés optiques des lames minces solides. Mémorial des Sciences Physiques. Gauthier-Villars, Paris, 1952.

[6] Bousquet, C. R. Acad. Sc., 1954, 238, 1485 et Thèse de Doctorat ès-Sciences, Paris, 29 février 1956. 